\title{
Effects of vitex agnes castus on hormonal imbalances in Polycystic Ovary Syndrome
}

\author{
Sugantha Saul*
}

Department of Biochemistry, T.B.M.L. College, Porayar 609 307, Tamilnadu, India

Received: 13 June 2017

Revised: 22 June 2017

Accepted: 07 July 2017

*Correspondence to:

Dr. Sugantha Saul,

Email: rsrrbethel@gmail.com

Copyright: (C) the author(s), publisher and licensee Medip Academy. This is an openaccess article distributed under the terms of the Creative Commons Attribution NonCommercial License, which permits unrestricted noncommercial use, distribution, and reproduction in any medium, provided the original work is properly cited.

\begin{abstract}
Background: The polycystic ovary syndrome (PCOS) is one of the most common endocrinopathies in women. Its incidence is assessed at 6-8\% of the female population in the reproductive age. It is caused by an imbalance of the female sex hormones and higher levels of male hormones called androgens.

Methods: In women with PCOS, the ovaries make more androgens than normal. High levels of these hormones affect the development and release of eggs during ovulation. Hence the hormonal imbalance were studied in three groups. In the present study ,8mg of estradiol valerate was used to induce PCOS in female albino rats. The levels of leutinizing hormone, follicle stimulating hormone, testosterone, dehydroepiandrosterone sulphate, Estradiol, Progesterone, and Prolaction were studied.

Results: The phytochemical analysis of vitex agnus castus was carried out and the positive effects of vitex agnus castus on the hormonal irregularities of PCOS were also studied. The present findings indicated that vitex agnus castus was found to be rich in phytochemicals.

Conclusions: The hormonal levels highly reflect the underlying hormonal imbalance in PCOS and the results obtained in the present study also proved that vitex agnus castus is more efficient in reversing the adverse effects of hormonal imbalance of PCOS.
\end{abstract}

Keywords: Hormonal imbalance, Polycystic ovary syndrome, Phytochemical analysis, Vitex agnus castus

\section{INTRODUCTION}

Polycystic ovarian syndrome in the most common endocrinopathy affecting approximately $6-10 \%$ of women in reproductive age. It is one of the leading causes of infertility resulting from chronic anovulation. It is a heterogenous condition characterized by chronic anovulation or infrequent ovulation, obesity, hirsutism, hyperandrogenism and numerous follicular cysts in enlarged ovaries. The syndrome is named for the tiny cysts that may form in the ovaries when hormone imbalance interrupts the ovulation process. Hence the ovaries have twice as many follicles than usual resulting in slightly larger than normal ovaries.

A main underlying problem with PCOS is a hormonal imbalance. PCOS is caused by an imbalance of the female sex hormone and higher levels of male hormones called androgens. Androgens are male hormones that females also make. In women with PCOS, the ovaries make more androgens than normal. High levels of these hormones affect the development and release of eggs during ovulation.

Vitex agnus castus (Verbenaceae), commonly referred to as chaste tree or chasteberry, is an aromatic flowering 
member of mint family. For thousands of years, vitex has been regarded as an important phytomedicine to treat gynecological disorders. Several potential mechanisms of action have been proposed to explain the activity of vitex agnus castus extracts, including inhibition of prolactin secretion, dopaminergic and estrogenic effects. ${ }^{1}$

A few clinical studies have also indicated that vitex agnus castus may also be a potential treatment for infertility due to hyperprolactinemia and luteal-phase defect, insufficient lactation, and to prevent miscarriages due to progesterone insufficiency. ${ }^{2}$ Vitex impacts key hormones that regulate and balance the menstrual cycle and to balance the levels and ratios of hormones required for normal menstrual function, ovulation and fertility. Vitex clearly demonstrated a decrease of estrogen effects and an increase of progesterone levels at normal doses. This effect was mediated by the pituitary gland. Vitex has been traditionally used to treat a number of ailments, but with particular emphasis on menstrual disorders and related hormonal problems.

The aim of the present study was to lighten the proposed hormonal disturbances for the development of PCOS and to study the beneficial roles of vitex agnus castus on the negative impacts of hormonal imbalances.

\section{METHODS}

\section{Preparation of plant extract}

The ripened vitex agnus castus fruits were harvested and were cleaned. They were dried in sunlight and were powdered. From the powder, the ethanol extract was prepared with $70 \%$ ethanol solvent for 6 hours at $35^{\circ} \mathrm{C}$ using a magnetic stirrer. The mixture was filtered through a Whatman No.1 filter paper. The resulting solution was evaporated under vacumm and then dried at $-50^{\circ} \mathrm{C}$ in a lyophiliser. The extract was stored in a freezer for further experiments. ${ }^{3}$ Phytochemical studies were carried out in the prepared extract.

\section{Animal models}

Female albino rats of Wistar strain approximately weighing 200-220g were used in this study. They were healthy animals purchased from the Indian Institute of Science, Bangalore. The animals were housed in spacious polypropylene cages bedded with rice husk. The animal room was well ventilated and maintained under standard experimental conditions (Temperature $27 \pm 2^{\circ} \mathrm{C}$ and 12 hour light/dark cycle) throughout the experimental period. All the animals were fed with standard pellet diet and water were provided ad libitum. They were acclimatized to the environment for one week prior to experimental use. The animal feed composition is crude protein $(22.3 \%)$, crude oil $(4.01 \%)$, crude fibre $(4.02 \%)$, Ash $(8.02 \%)$ and sand silica $(1.02 \%)$. All animal experiments were performed according to the ethical guidelines suggested by the
Institutional Animal Ethics Committee (IAEC) in the laboratory.

\section{Induction of PCOS}

Estradiol valerate $(\mathrm{EV})$ is an effective drug used to induce PCOS in rodents. ${ }^{4}$ In the present study PCOS was induced by the single intramuscular injection of $8 \mathrm{mg}$ of $\mathrm{EV}$ in the female albino rats.

This dose induces persistent estrous and the rats ceased ovulation and developed characteristics of human PCOS, including large cystic follicles in the ovaries and induce the hormonal alterations of PCOS.

\section{Design of the study}

The control and EV induced PCOS rats were subdivided into 3 groups with 7 animals in each group.

Group 1: Normal control group

Rats fed with standard pellet diet and water with normal ovaries.

\section{Group 2: PCOS induced rats}

The rats fed with normal pellet diet were induced with an intramuscular injection of $8 \mathrm{mg}$ of $\mathrm{EV}$ and allowed to live under normal conditions for 30 days. On the $30^{\text {th }}$ day, they were sacrificed under chloroform anesthesia.

Group 3: PCOS induced rats treated with vitex agnus castus extract

The PCOS induced rats were treated with vitex agnus castus $(250 \mathrm{mg} / \mathrm{kg}$ body weight $/ 5 \mathrm{ml}$ double deionised water / day) orally from the next day of induction for 30 days.

\section{Collection of blood and preparation of serum sample}

On completion of the experimental period, animals were anaesthetized with chloroform prior to dissection. Blood was collected by cardiac puncture into serum separator tubes. The blood was allowed to clot by standing at room temperature for 30 minutes and then refrigerated for another 30 minute. The resultant clear part was centrifuged at 3000rpm for 10 minutes, and then the serum (supernatant) was isolated and stored at refrigerated until required for analysis.

\section{Statistical analysis}

Statistical analyses were performed using the statistical package for social sciences 12.0 (SPSS 12.0). Values are reported as mean \pm SD. Statistical significance was attributed to $\mathrm{P}<0.01$. 


\section{RESULTS}

\section{Screening of phytochemicals}

The qualitative analysis of vitex agnus castus showed the presence of polyphenols, saponins, tannins, steroids, terpenoids and triterpenoids.

\section{Hormonal assay}

The hormonal imbalance is associated with the classic form of PCOS. In order to find out its negative effects on the development of PCOS, the levels of reproductive hormones were studied in all groups.

\section{Leutinizing hormone (LH) and Follicle stimulating} hormone (FSH)

The level of LH was highly elevated in EV control group $(0.27 \pm 0.02)$ when compared to control group $(0.13 \pm 0.02)$. But there was no significant difference in the level of FSH between the study groups. This inappropriate $\mathrm{LH}$ and FSH secretion lead to the abnormal LH/FSH ratio which is one of the characteristic features of PCOS. This elevated level was gradually decreased in rats treated with herbal drug in group III $(0.15 \pm 0.02)$ and were statistically significant (p $<0.001$ ) (Figure 1). The highest $\mathrm{LH} / \mathrm{FSH}$ ratio was observed in EV control group with the ratio of 2.4. This ratio was almost reversed and attained the ratio of 1.0 in rats treated with vitex agnus castus.

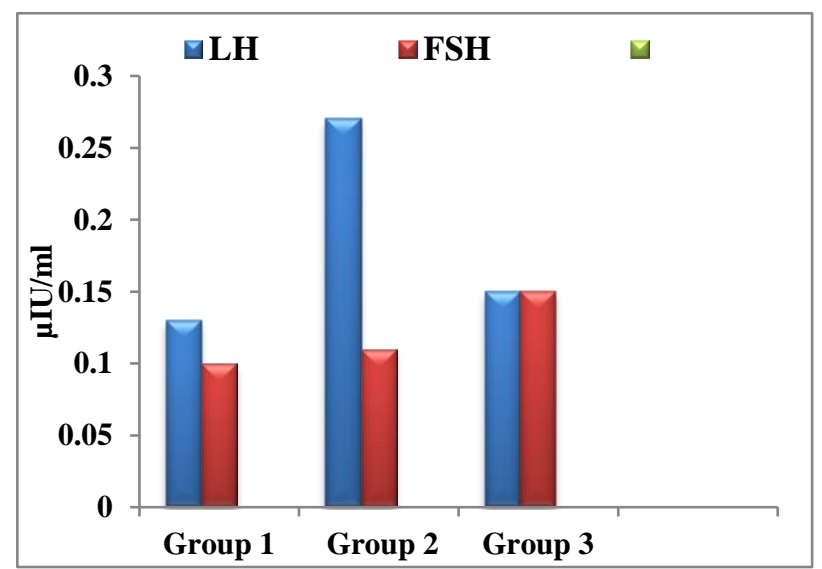

Figure 1: The levels of LH and FSH between control and study groups.

A long acting estradiol valerate (EV) has been used for the experimental induction of PCO. ${ }^{4,5}$ Female rats exhibit anovulatory acyclicity, persistent vaginal cornification and polycystic ovaries, 4 weeks after a single treatment with estradiol valerate $(\mathrm{EV})$.

\section{Testosterone (TST) and Dehydroepiandrosterone sulphate (DHEAS)}

The levels of testosterone and DHEAS was most prominent in PCOS induced rats which is a characteristic feature of hyperandrogenism. The levels of testosterone $(1.88 \pm 0.23)$ (Figure 2) and DHEAS (245.85 \pm 4.09$)$ in group II was increased when compared to control and it was highly significant $(\mathrm{p}<0.001)$. This relative increase in unbound concentrations of DHEAS and testosterone is clinically related with hyperandrogenism of hirsutism. ${ }^{6,7}$

Herbal therapy was highly effective in controlling the levels of testosterone and DHEAS. This was reflected in the levels of testosterone $(0.95 \pm 0.17)$ and DHEAS $(218.28 \pm 2.13)$ in group III and it was highly significant ( $p$ $<0.001)$.

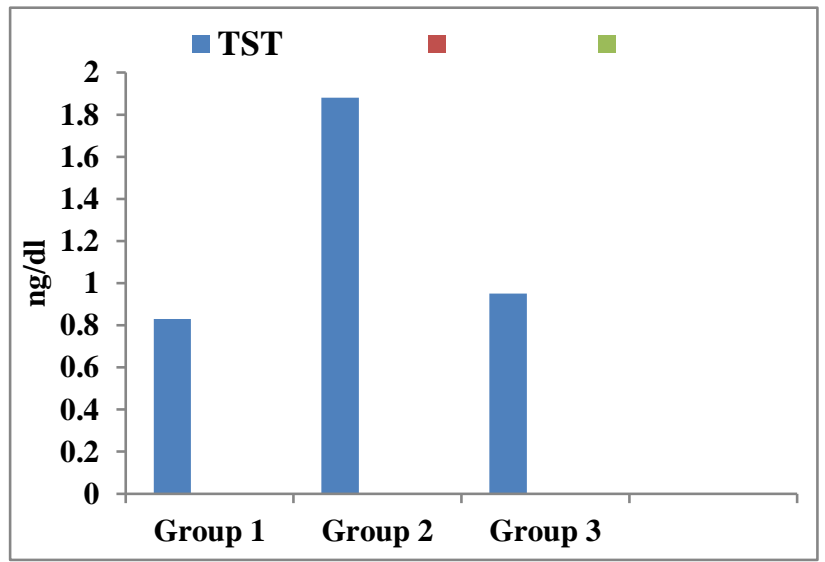

Figure 2: The levels of TST between control and study groups.

Table 1: The hormonal profiles between control and study groups.

\begin{tabular}{|llll|}
\hline Variables & 1 & 2 & $\mathbf{3}$ \\
\hline $\begin{array}{l}\mathrm{LH} \\
(\mu \mathrm{IU} / \mathrm{ml})\end{array}$ & $0.13 \pm 0.02$ & $0.27 \pm 0.02$ & $0.15 \pm 0.02$ \\
\hline $\begin{array}{l}\mathrm{FSH} \\
(\mu \mathrm{IU} / \mathrm{ml})\end{array}$ & $0.10 \pm 0.03$ & $0.11 \pm 0.03$ & $0.15 \pm 0.03$ \\
\hline TST(ng/dl) & $0.83 \pm 0.07$ & $1.88 \pm 0.23$ & $0.95 \pm 0.17$ \\
\hline $\begin{array}{l}\mathrm{ESD} \\
(\mathrm{pg} / \mathrm{ml})\end{array}$ & $78.37 \pm 0.83$ & $94.31 \pm 0.68$ & $81.05 \pm 0.60$ \\
\hline $\begin{array}{l}\text { PGT } \\
(\mathrm{pg} / \mathrm{ml})\end{array}$ & $7.33 \pm 0.2$ & $3.9 \pm 0.24$ & $7.14 \pm 0.3$ \\
\hline $\begin{array}{l}\mathrm{DHEAS} \\
(\mu \mathrm{g} / \mathrm{dl})\end{array}$ & $212.28 \pm 3.35$ & $245.85 \pm 4.09$ & $212.28 \pm 2.13$ \\
\hline $\begin{array}{l}\mathrm{PRL} \\
(\mathrm{ng} / \mathrm{dl})\end{array}$ & $10.95 \pm 0.81$ & $21.85 \pm 0.59$ & $12.03 \pm 0.92 \mathrm{~s}$ \\
\hline $\mathrm{LH} / \mathrm{FSH}$ & 1.3 & 2.4 & 1.0 \\
\hline
\end{tabular}

\section{Estradiol (ESD) and Progesterone (PGT)}

The underlying hormonal imbalance also exists in the levels of estradiol. The level of estradiol was highly significant in group II $(94.31 \pm 0.68)$ in comparison with the control group $(78.37 \pm 0.83)$ but was significantly decreased in group III $(81.05 \pm 0.60)$ and was highly significant $(\mathrm{p}<0.001)$. 
Decreased level of progesterone was also noticed in group II rats $(3.9 \pm 0.24)$ and Vitex agnus castus was highly effective in significantly increasing the level of progesterone in group III (7.14 \pm 0.33$)$ (Figure 3). These results clearly evident that the vitex agnus castus was efficient to overcome the luteal insufficiency of progesterone.

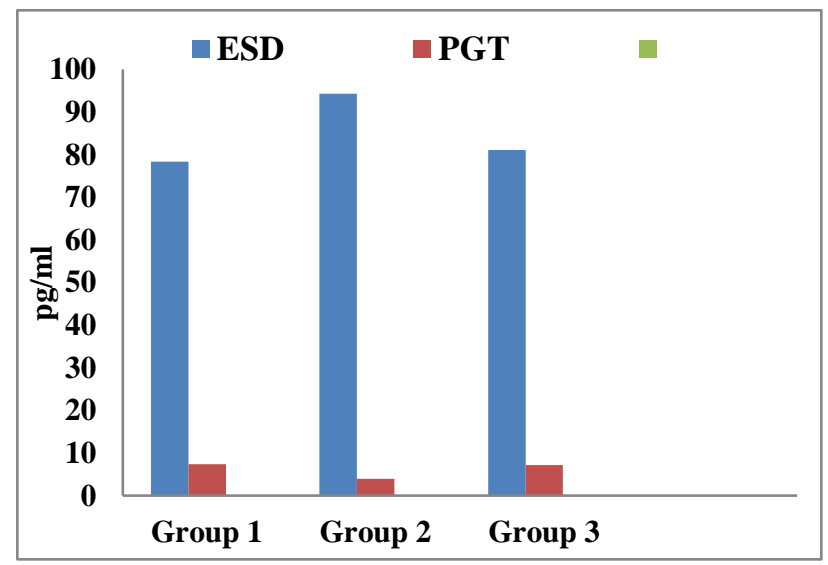

Figure 3: The levels of ESD and PGT between control and study groups.

\section{Prolaction (PRL)}

PCOS induced rats showed high levels of prolactin (21.85 \pm 0.59$)$ in comparison with control rats $(10.95 \pm 0.81)$ but a significant reduction was noticed by herbal treatment in group III $(12.03 \pm 0.92)$. and was highly significant $(\mathrm{p}<0.001)$.

The clinical studies have also indicated that vitex agnus castus may be a potential treatment for infertility due to hyperprolactinemia and luteal phase defect. ${ }^{8,9}$

\section{DISCUSSION}

The presence of phytochemicals in vitex agnus castus reflected the complex mixture of total phenolic and flavanoid compounds. Studies have indicated that the phytochemicals as flavanoids, carotenoids and other phenolic compounds provide significant antioxidant activity and health benefit. This could account for the high antioxidant activity of vitex agnus castus and thus used as a naturally potential antioxidant source. ${ }^{10}$

The present study showed marked changes in the hormonal levels of $\mathrm{EV}$ induced rats and reflected the characteristic features of PCOS. Even though there was no change in FSH level of group II rats, the LH levels were significantly increased. After EV treatment, the LH level increases to very high concentrations for the follicles to expand and stabilize as cysts. ${ }^{11}$ Vitex agnus castus significantly reduce the LH levels which reversed the LH/FSH ratio, promoting ovulation in PCO rats. The present findings of the effect of vitex agnus castus on the levels of LH in PCOS, support recent findings that states vitex has been traditionally used to treat fibroid cysts and infertility. ${ }^{12}$

The levels of TST and DHEAS in group III reflected the direct effect of vitex agnus castus on the inhibition of androgen production in human thecal cells. The present results were in side with Amann, who have treated the vitex agnus castus extract for hormonally induced acne and this may be due to normalization of steroid hormones and the restoration of ovulation. ${ }^{13}$ Since hyperprolactinemia is positively correlated with hyper secretion of adrenal androgens, the positive effects of vitex agnus castus on the increased levels of prolactin could also account for this anti androgenic effects. ${ }^{14}$

In the present findings, the increased levels of estradiol and decreased level of progesterone results in failure of ovulation which is one of the notable features of infertility. But these altered levels are reversed in rats treated with vitex agnus castus fruit extract for the normalization of progesterone concentrations. Several groups have also demonstrated that vitex agnus castus extracts bind to the estrogen receptor and have weak estragenic effects, suggesting that chasteberry may also affect the estrogen/progesterone balance. ${ }^{15}$ In the present study, high levels of prolaction was also controlled in rats treated with vitex agnus castus. Extreme levels of prolactin may lead to amenorrhea and this can be treated by the extracts of vitex agnus castus and this was evidenced earlier itself to treat the gynecological disorders such as hypermenorrhea and secondary amenorrhea with the extracts of vitex agnus castus.

\section{CONCLUSION}

The results evidenced the underlying hormonal disturbances of PCOS and these act as the causative agents for the endocrine abnormalities of PCOS. The present study also reflected the ameliorating efficacy of vitex agnus castus on the hormonal changes of PCOS.

\section{Funding: No funding sources \\ Conflict of interest: None declared}

Ethical approval: The study was approved by the Institutional Animal Ethics Committee

\section{REFERENCES}

1. Wuttke W, Jarry H, Christoffel V, Spengler B, Seidlova WD. Chaste tree (Vitex agnus castus). Pharmacological and clinical indications, Phytomedicine. 2003;10:348-57.

2. Gerhard I, Patek A, Monga B, Blank A. Gilling-Smith C, Story H, Rogers V, Franks S. Evidence for a primary abnormality of thecal cell steroidogenesis in the polycystic ovary syndrome. Clin Endocrinol. 1997a;47(1):93-9.

3. Gulcin I. The antioxidant and radical scavenging activities of black pepper seed. J. Food Sci. Nutr. 2005;56(7):491-9. 
4. Brawer JR, Munoz M, Farookhi R. Development of the polycystic ovarian condition(PCO) in the estradiol valerate-treated rat. Biol. Reprod. 1986;35:647-55.

5. Brawer JR, Naftolin J, Martin J, Sonnenschein C. Effects if a single injection of estradiol valerate on the hypothalamic arcuate nucleus and on reproductive function in the female rat. Endocrinology. 1978;103:501-12.

6. Baird DT, Corker CS, Davidson DW, Hunter WM, Michie EA, Van Look PF. Pituitary-ovarian relationships in polycystic ovary syndrome. J. Clin. Endocrinol. Metab. 1977;45:798-801.

7. McKenna TJ. Pathogenesis and treatment of polycystic ovary syndrome. $\mathrm{N}$ Engl $\mathrm{J}$ Med. 1988;318:558-62.

8. Merz PG, Gorkow C, Schroedter A, Rietborch S, Sieder C, Loew D, et al. The effects of a special Agnus castus extract (BP1095E1) on prolactin secretion in healthy male subjects. Exp. Clin. Endocrinol. Diab. 1996;104:447-53.

9. Gerhard I, Patek A, Monga B, Blank A, Gilling-Smith $\mathrm{C}$, Story H, et al. Evidence for a primary abnormality of thecal cell steroidogenesis in the polycystic ovary syndrome. Clin Endocrinol. 1997a;47(1):93-9.

10. Hadju A, Hohmann J, Forgo P, Martinek T, Dervarics M, Zupko I, et al. Deterpenoids and flavonoids from the fruits of vitex agnus castus and antioxidant activity of the fruit extracts and their constituents. Phytother. Res. 2007;21(4):391-4.

11. Welschen R. Amounts of gonadotropins required for normal follicular growth in hypohysecomized adult rats. Act Endocrinol. 1973;72:137-55.

12. McGuffin M, Hobbs C, Upton R, Goldberg A. Botanical Safety Handbook; CRC Press: Boca Raton; 1997:231.

13. Amann W. Acne vulgaris and Agnus castus (Agnolyt ${ }^{\circledR}$ ). Z. Allgemeinmed. 1975;51:1645-8.

14. Agbaht K, Yerlikaya H, Demir O, Gullu S. Hyperprolactenemia in polycystic ovary syndrome. Endocrine Abstracts. 2009;20:653.

15. Yi L, Lilan L, Haibo Z. Levels of lipid perioxides and superoxide dismutase in peritoneal fluid of patients with endometriosis. Journal of Huazhong University of Science and Technology. Medical Sciences. 2001 Jun 1;21(2):166-7.

Cite this article as: Saul S. Effects of vitex agnes castus on hormonal imbalances in Polycystic Ovary Syndrome. Int J Basic Clin Pharmacol 2017;6:20515. 